\title{
Infinite geometric frustration in a cubic dipole cluster
}

\author{
Johannes Schönke, ${ }^{1}$ Tobias M. Schneider, ${ }^{1,2}$ and Ingo Rehberg ${ }^{3}$ \\ ${ }^{1}$ MPRG-ECPS, MPI für Dynamik und Selbstorganisation, Am Faßberg 17, 37077 Göttingen, Germany \\ ${ }^{2}$ Emergent Complexity in Physical Systems Laboratory (ECPS), Ecole Polytechnique Fédérale de Lausanne, Switzerland \\ ${ }^{3}$ Physikalisches Institut, Experimentalphysik V, Universität Bayreuth, 95440 Bayreuth, Germany \\ (Received 24 July 2014; revised manuscript received 5 January 2015; published 29 January 2015)
}

\begin{abstract}
The geometric arrangement of interacting (magnetic) dipoles is a question of fundamental importance in physics, chemistry, and engineering. Motivated by recent progress concerning the self-assembly of magnetic structures, the equilibrium orientation of eight interacting dipoles in a cubic cluster is investigated in detail. Instead of discrete equilibria we find a type of ground state consisting of infinitely many orientations. This continuum of energetically degenerate states represents a yet unknown form of magnetic frustration. The corresponding dipole rotations in the flat potential valley of this Goldstone mode enable the construction of frictionless magnetic couplings. Using computer-assisted algebraic geometry methods, we moreover completely enumerate all equilibrium configurations. The seemingly simple cubic system allows for exactly 9536 unstable discrete equilibria falling into 183 distinct energy families.
\end{abstract}

DOI: 10.1103/PhysRevB.91.020410

PACS number(s): 75.10.-b, 05.65.+b, 41.20.Gz

Magnetism has fascinated mankind for millenia [1]. Today, even the smallest magnets can hardly be overestimated in their relevance for magnetic storage technology. A fascinating example for the interplay of magnetic particles is their self-arrangement in cubic lattice clusters (see, e.g., [2,3]). Its macroscopic analog is the toy known as "magnetic cube puzzle" shown in Fig. 1(a), a stable arrangement of spherical magnets in a simple cubic cluster. How are these magnetic spheres oriented in such an ordered cluster? For the minimal arrangement within this class, namely, a cube consisting of eight magnets [see Fig. 1(b)], the answer is intriguing: There are infinitely many orientations. We find the ground state to be a continuum of energetically degenerate states - an extreme form of magnetic frustration. The phenomenon of frustration arises when the system cannot simultaneously minimize all dipole-dipole interaction energies (see [4] for a recent review). As this continuum is the ground state of the cube system, the question arises: Are there any other equilibrium orientations? Through our application of methods from numerical algebraic geometry (see Supplementary 4 [5]) we are able to construct and classify the complete set of equilibrium states. This set comprises thousands of unstable discrete dipole orientations in addition to the continuous states. We stress here that we find all equilibrium configurations (stable and unstable) unlike commonly used relaxation methods.

The study of equilibrium states of dipolar hard and soft spheres has a long history in the context of magnetic colloids. Early works [6-8] on these so-called ferrofluids investigate the phase behavior (colloidal crystal structures, chain formation, string fluids, etc.). More recently, thermodynamic properties of two-dimensional (2D) monolayer systems have been studied [9] and a full phase diagram in terms of dipole strength and packing fraction was given [10]. Further, the self-assembly and transition from rings to chains controlled by an external field was investigated (see, e.g., [11-13]). Isolated planar dipole clusters in external fields have been considered in quadratic and ringlike configurations [14,15], as well as the ground states of planar chains and rings and their interaction [16]. A three-dimensional (3D) isolated cluster of uniformly distributed dipoles on a sphere is given in [17] and equilibrium configurations with a hierarchy of chains, rings, and tubes have been found in [18] and further detailed in [19]. In all these related works, a continuous equilibrium (ground) state of a dipole cluster has not been discovered. Further, we characterize the complete set of all possible equilibrium configurations of a cluster.

The precise problem addressed reads as follows: $N$ freely orientable dipoles of equal magnitudes are given together with their fixed positions in space. Which equilibrium configurations are possible? How many of these equilibria are stable? Which equilibrium represents the energetically favorable ground state, i.e., has the lowest energy? Here, we consider the classical dipole-dipole interaction with the magnetic energy per dipole,

$$
E=\frac{1}{N} \sum_{i<j}^{N} \frac{\mathbf{m}_{i} \cdot \mathbf{m}_{j}-3\left(\mathbf{m}_{i} \cdot \mathbf{e}_{i j}\right)\left(\mathbf{m}_{j} \cdot \mathbf{e}_{i j}\right)}{\left|\mathbf{r}_{i j}\right|^{3}},
$$

where $\mathbf{m}_{1}, \ldots, \mathbf{m}_{N}$ are the variable dipole moments with equal magnitudes $\left|\mathbf{m}_{i}\right|=1$, and $\mathbf{r}_{i j}$ denotes the fixed relative position vector between dipole $i$ and $j$ with $\mathbf{e}_{i j}$ being the
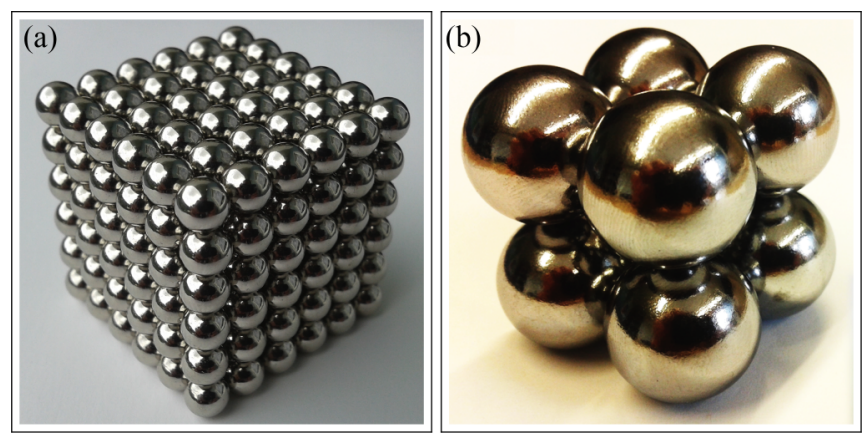

FIG. 1. (Color online) (a) The "magnetic cube puzzle": A stable arrangement of 216 spherical magnets in a $6 \times 6 \times 6$ simple cubic cluster. (b) The minimal simple cubic configuration of spherical magnets, in a $2 \times 2 \times 2$ cluster. The ground state of this arrangement is not a single equilibrium configuration with only one discrete orientation for each dipole but rather a continuum of infinitely many configurations. 

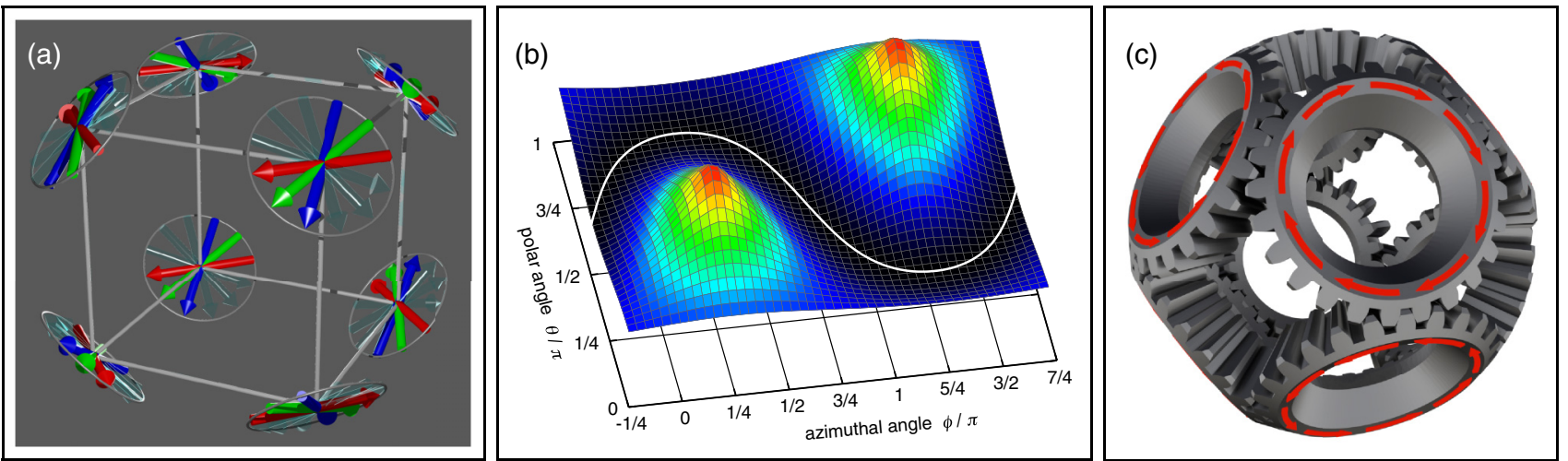

FIG. 2. (Color online) (a) Rendering of eight dipoles as arrows located at the corners of a cube. The colored and translucent arrows show selected orientations in the ground state of the system which is a continuum of infinitely many configurations. The continuum corresponds to rotations of the dipoles in planes perpendicular to the cube's volume diagonal of the respective corner in analogy to the mechanical system in Fig. 2(c). Consecutive arrows are seperated by $30^{\circ}$. Along the continuum we repeatedly pass through two particularly notable configurations: First, the color red shows two counter-rotating rings of four dipoles each lying in the upper and lower faces of the cube. Secondly, the color green shows two groups of four dipoles lying in two diagonal planes perpendicular to each other. Thirdly, the color blue shows two rings similar to red but now in the left and right faces of the cube. Pairs of dipoles in opposing corners are always parallel. (b) Magnetic energy landscape for eight dipoles located at the corners of a cube. The energy is shown as a function of the two orientation angles of one dipole, covering all possible orientations. The other seven dipoles adjust to the respective minimum energy configuration. The white line through the "valley" marks the continuous ground state. (c) Rendering of eight bevel gears located at the corners of a cube. The rotation axes point to the cube's center and every gear interlocks with its three edge neighbors. The motion of this mechanical system, indicated by the red arrows, corresponds to the motion of the ground state continuum in the magnetic system shown in Figs. 1(b) and 2(a). This analogy opens up new engineering possibilities to construct frictionless magnetic couplings.

corresponding unit vector (cf. Supplementary 1 [5]). The equilibrium condition $\nabla E=0$ corresponding to stationary points in the energy landscape is represented by a set of strongly coupled polynomial equations for all dipole orientations of the cluster (cf. Supplementary 2 [5]). Numerical algebraic geometry methods described in Supplementary 4 [5] allow one to construct the complete solution set and thereby find all equilibrium configurations.

We investigate different elementary clusters of dipoles (see below) but report here in detail about one case which proves to be special: the case of eight dipoles located at the corners of a cube. As pointed out before, the ground state of this arrangement is not a single configuration with only one discrete orientation for each dipole but rather a continuum of infinitely many configurations. Below we refer to this ground state continuum in short as the "continuum"; its spatial structure is shown in Fig. 2(a). The continuum exhibits a reflection symmetry through the three central planes (each parallel to a pair of cube faces) - the dipole moments (as axial vectors) flip sign under reflection. If we rotate one of the dipoles along the continuum all the other dipoles rotate accordingly. Such a rotation is not affected by any magnetic counterforce since we stay on the same level in the magnetic energy landscape. This walk through the "ground state valley" is depicted in Fig. 2(b). For the unit cube the energy of the continuum has the characteristic value of $E_{c}=-2+\sqrt{2} / 16+\sqrt{3} / 18$ and its net magnetic moment is zero. Furthermore, the toroid moment with respect to the center of the cube is also zero (cf. Supplementary 1 [5]).

The continuum described here sheds a new light on frustration in magnetic systems, which has regained a lot of attention in recent years because of tailor-designed structures (e.g., "artificial spin ice") showing new and exciting thermodynamic behavior [4]. Classically, frustration has been studied with the following assumptions: Discrete spins interact (anti)ferromagnetically with nearest neighbors, mostly on an infinite lattice, though some interesting studies on finite isolated magnetic clusters do also exist (e.g., [20]). In these discrete systems frustration emerges necessarily from a countable number of different states which are energetically degenerated. Furthermore, there are quantum mechanical models of 2D spin systems ( $X Y$ model) with "antidipolar" interactions [21] (the Hamiltonian has the opposite sign compared to the dipolar interaction) or general bilinear spin interactions [22] (which would include dipolar interactions but they are not considered in [22]). These models can possess continuous states in tetrahedral arrangements [21,23] or on the pyrochlore lattice [22]. In contrast to all these systems we study classical dipoles which are freely orientable in $3 \mathrm{D}$ space subject to not only nearest-neighbor but fully coupled interactions in a finite system of $N$ dipoles. For dipolar interactions, the resulting type of frustration in the cube has a new quality: The finite system reported here has an uncountable infinite number of different states which are energetically degenerated. In a sense, it is therefore "infinitely frustrated." Note that the continuous state is not a simple consequence of the individual dipoles being freely (continuously) orientable. In general, the anisotropic dipole-dipole interaction induces discrete equilibrium configurations (see examples below) so that the cube continuum is indeed exceptional.

A further intriguing aspect of the continuum is the existence of an exact mechanical analog. The aforementioned possibility to rotate one dipole along the continuum with the other dipoles following accordingly, raises the question: Can we reproduce 
the same dynamics through another type of interaction? It is possible with eight bevel gears, as explained in Fig. 2(c). The analogy between the mechanical and the magnetic system allows for new ways to engineer couplings. Since there are no magnetic counterforces to overcome along the continuum, one can build a frictionless sevenfold magnetic coupling. Practical applications require robustness of the ground state continuum with respect to perturbations of the perfect cubic arrangement as well as to deviations from the point dipole approximation. Experimental tests for two prototype realizations directly confirm the robustness of our results. Details about these constructions together with the analysis for the perturbed arrangement are given in Supplementary 6 [5]).

The cube continuum is embedded in a richly structured state space which contains a multitude of other equilibrium configurations. We now provide the complete enumeration for all possible equilibria in the cube. This is a highly nontrivial problem since we aim at determining all solutions of a strongly coupled system of 32 polynomial equations (cf. Supplementary 2 [5]). The number of possible zero-dimensional (0D) solutions (corresponding to discrete isolated orientations) grows exponentially with the number of dipoles. For the cube we have a simple upper bound of $2^{24}=16777216$ possible 0D solutions (cf. Supplementary 3 for the derivation [5]). Additionally, there may be higher dimensional solution manifolds; the continuum described above is a $1 \mathrm{D}$ example. Fortunately, this system size can be tackled with methods known under the term "numerical algebraic geometry" which were developed in the last two decades [24-26] (Supplementary 4 [5]). The result is an astonishing number of 1594032 (generally complex) OD solutions. Besides that, there are four 1D continua, two of them being complex, plus the ground state continuum described above and a second real continuum. Higher dimensional solution manifolds do not exist (cf. Supplementary 4 [5]).

Extracting the physically meaningful real-valued subset of OD solutions, we still end up with 9536 solutions. These can now be sorted into energy families, i.e., all solutions with identical energies belong to the same family. There are always at least two configurations with identical energy because the polarity symmetry (reversing the orientation of all dipoles) is again a solution and leaves the energy unchanged. In addition, there is the full symmetry group of the cube of order 48 . Depending on the symmetries of the respective solution, we therefore may have up to $2 \times 48=96$ members in one energy family. In general, there could be more members in a family if two configurations which are not related through symmetries have accidentally the same energy, although this does not happen in the cube. The sorting gives rise to 183 families of OD solutions. The energy spectrum of these families together with some exemplary configurations is shown in Fig. 3. An interactive web gallery of all equilibrium configurations in the cube can be found in [27].

The stability of any equilibrium configuration in our system is determined by the $2 N$ eigenvalues $\lambda_{k}$ of the Hessian matrix $\mathbf{H}$, i.e., the matrix of all second-order partial derivatives of the energy $E$ with respect to the $2 N$ degrees of freedom. A general result for systems considered here is the relation

$$
\sum_{k=1}^{2 N} \lambda_{k}=\operatorname{Tr}(\mathbf{H})=-4 E
$$

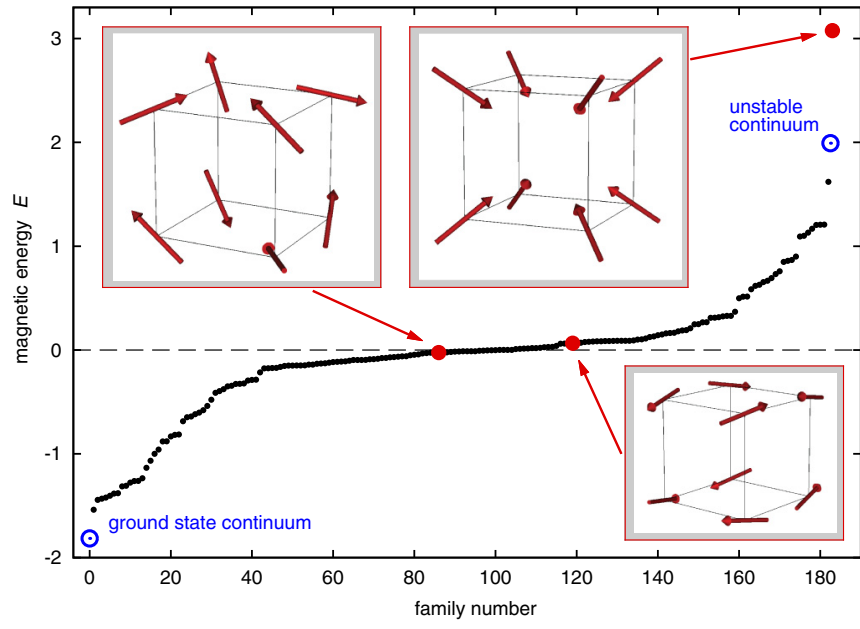

FIG. 3. (Color online) Magnetic energy spectrum of all 183 families of discrete equilibrium configurations for eight dipoles located at the corners of a cube. Each family (black filled circles) with its unique energy may contain up to 96 members due to polarity and cube symmetries. The red filled circles mark positions of exemplarily chosen configurations which are displayed in their respective inset. The upper left inset shows a fully unstructured configuration with no apparent symmetries, therefore the family has 96 members. The lower right inset shows a more structured configuration with some obvious symmetries, so this family has only six distinguishable members. The upper right inset shows the maximum energy configuration which is the most ordered one with all dipoles oriented to the cube center; all symmetries of the cube are retained and only the polarity flip gives a new configuration, so this family has two members. The additional two blue circles mark the energetic positions of the ground state continuum and the second real continuum.

(see Supplementary 5 for the derivation [5]). It shows that a positive energy $E>0$ is a sufficient condition for instability. Since the sum of all eigenvalues is negative, there must be at least one negative $\lambda_{k}$, which classifies a configuration as unstable. From this we can conclude that a negative energy $E<0$ is a necessary condition for stability. For the cube this means that the second real continuum is unstable (cf. Fig. 3). Calculating the eigenvalues, we actually find all 183 families of discrete equilibria to be (unstable) saddles in the energy landscape, i.e., mixed positive and negative $\lambda_{k}$. The only exception is the maximum energy family which is necessarily unstable in any direction. This confirms that the ground state continuum is the only stable state.

Now we put the cube and its continuum into the context of other regular dipole clusters. We consider the dipoles to be located at the vertices of various regular geometric shapes. Table I lists the number of solutions and energy families for different arrangements. The ground state continuum of the cube seems to be an exceptional property. So far we did not find any other regular arrangement which has this feature. Simple planar arrangements like the line segment or the equilateral triangle do not have continua; their ground states are necessarily discrete. The two smaller (in terms of numbers of corners) platonic solids, i.e., tetrahedron and octahedron, have continua, but these are unstable (see also [23]). Therefore, 
TABLE I. Number of discrete equilibrium configurations (DEC) for different arrangements of freely orientable dipoles. The dipoles are positioned at the corners of the respective arrangement. $N$ is the number of dipoles, $S_{\max }$ is a simple upper bound (cf. Supplementary 3 [5]) for the number of possible DECs, $S$ is the actual number of (generally complex) DECs, $S_{\text {real }}$ is the number of real-valued DECs, and $F_{\text {real }}$ is the number of energy families the real-valued DECs split into. The last row serves solely as an illustration of exponential complexity.

\begin{tabular}{lccccc}
\hline \hline Arrangement & $N$ & $S_{\max }=2^{3 N}$ & $S$ & $S_{\text {real }}$ & $F_{\text {real }}$ \\
\hline Line segment & 2 & 64 & 8 & 8 & 4 \\
Triangle & 3 & 512 & 96 & 48 & 8 \\
Tetrahedron & 4 & 4096 & 420 & 116 & 10 \\
Octahedron & 6 & 262144 & 37608 & 1156 & 43 \\
Cube & 8 & 16777216 & 1594032 & 9536 & 183 \\
Icosahedron & 12 & 68719476736 & $?$ & $?$ & $?$ \\
\hline \hline
\end{tabular}

their ground states are also discrete. Another common feature of the regular arrangements investigated is the existence of only one stable configuration (modulo energetic degeneracies due to symmetries). For larger clusters, we expect several stable configurations to coexist.
In this study we report on a yet unknown type of ground state for systems of interacting dipoles-a continuum of infinitely many energetically degenerate orientations. This result raises several new questions: Is the cube the only cluster that admits a stable (possibly ground state) continuum, allowing any number of dipoles in any arrangement? What happens to a continuum in an external field? What is the susceptibility of an arrangement possessing a continuum? What consequences do (stable or unstable) continua have for the dynamics of magnetic clusters, or more general, for their thermodynamic properties? The thermodynamic stability is especially relevant for the miniaturization of domains in magnetic information storage, specifically in the extreme limit of nanostructured systems (see, e.g., [28]). The height of energetic barriers between different coding states limits the thermodynamic long-term stability. In our case the completely vanishing energy barrier of the continuum prevents any information storage.

The authors are grateful to Michael Grunwald [29] for the design and construction of the 3D printed dipole cube shown in Supplementary 6 [5] and for the rendering of Fig. 2(c). J.S. thanks Hecke Schrobsdorff for an introduction to POV-Ray and Priya Subramanian for numerous fruitful distractions.
[1] Platon, Ion, section 533d (413 BCE).

[2] A. Ahniyaz, Y. Sakamoto, and L. Bergström, Proc. Natl. Acad. Sci. USA 104, 17570 (2007).

[3] S. Förster, T. Friedrich, S. Mehdizadeh Taheri, I. Rehberg, and S. Rosenfeldt, Book of Abstracts of the 13th German Ferrofluid Workshop, Benediktbeuern, http://www.ferrofluidverein.de/

[4] C. Nisoli, R. Moessner, and P. Schiffer, Rev. Mod. Phys. 85, 1473 (2013).

[5] See Supplemental Material at http://link.aps.org/supplemental/ 10.1103/PhysRevB.91.020410 for a detailed description of how to derive the equilibrium equations in a form suitable for numerical solution and some details about the software tools used to solve these equations. Further, proofs for the upper bound of isolated equilibria and for the stability criterion are given. Finally, two experimental realizations and a robustness analysis are presented.

[6] J. J. Weis and D. Levesque, Phys. Rev. Lett. 71, 2729 (1993).

[7] J. J. Weis and D. Levesque, Phys. Rev. E 48, 3728 (1993).

[8] P. I. C. Teixeira, J. M. Tavares, and M. M. T. da Gama, J. Phys.: Condens. Matter 12, R411 (2000).

[9] H. Morimoto, T. Maekawa, and Y. Matsumoto, Phys. Rev. E 68, 061505 (2003).

[10] A.-P. Hynninen and M. Dijkstra, Phys. Rev. Lett. 94, 138303 (2005).

[11] P. Jund, S. G. Kim, D. Tománek, and J. Hetherington, Phys. Rev. Lett. 74, 3049 (1995).

[12] D. Tománek, S. Kim, P. Jund, P. Borrmann, H. Stamerjohanns, and E. R. Hilf, Z. Phys. D: At., Mol. Clusters 40, 539 (1997).
[13] F. Kun, W. Wen, K. F. Pál, and K. N. Tu, Phys. Rev. E 64, 061503 (2001).

[14] P. Melenev, V. Rusakov, and Y. Raikher, J. Magn. Magn. Mater. 300, e187 (2006).

[15] Y. A. Koksharov, G. Khomutov, E. Soldatov, D. Suyatin, I. Maximov, L. Montelius, and P. Carlberg, Thin Solid Films 515, 731 (2006).

[16] T. A. Prokopieva, V. A. Danilov, S. S. Kantorovich, and C. Holm, Phys. Rev. E 80, 031404 (2009).

[17] P. Melenev, V. Rusakov, and Y. Raikher, Tech. Phys. Lett. 34, 248 (2008).

[18] A. S. Clarke and G. N. Patey, J. Chem. Phys. 100, 2213 (1994).

[19] R. Messina, L. A. Khalil, and I. Stanković, Phys. Rev. E 89, 011202 (2014).

[20] E. Mengotti, L. J. Heyderman, A. Fraile Rodríguez, A. Bisig, L. Le Guyader, F. Nolting, and H. B. Braun, Phys. Rev. B 78, 144402 (2008).

[21] M. E. Zhitomirsky, M. V. Gvozdikova, P. C. W. Holdsworth, and R. Moessner, Phys. Rev. Lett. 109, 077204 (2012).

[22] L. Savary, K. A. Ross, B. D. Gaulin, J. P. C. Ruff, and L. Balents, Phys. Rev. Lett. 109, 167201 (2012).

[23] It is interesting to note that for the tetrahedral arrangement we find an unstable continuous state (not discussed in this Rapid Communication) which is most probably related to the continuous state in [21]. Since our state is a saddle in the energy landscape, it remains an (unstable) saddle for the opposite sign in the Hamiltonian. Therefore, the stability of this state in [21] must follow from the restriction to the $X Y$ model. 
[24] A. J. Sommese and J. Verschelde, J. Complexity 16, 572 (2000). [25] J. D. Hauenstein, A. J. Sommese, and C. W. Wampler, Appl. Math. Comput. 218, 1240 (2011).

[26] D. J. Bates, J. D. Hauenstein, A. J. Sommese, and C. W. Wampler, Numerically Solving Polynomial Systems with Bertini (SIAM, Philadelphia, 2013).
[27] J. Schönke, An interactive gallery of the dipole cube, http://ecps.ds.mpg.de/people/cube_gallery/cube_gallery.html

[28] N. Romming, C. Hanneken, M. Menzel, J. E. Bickel, B. Wolter, K. von Bergmann, A. Kubetzka, and R. Wiesendanger, Science 341, 636 (2013).

[29] M. Grunwald, http://audiots.wordpress.com/ 